\title{
Magnetization of Nanoparticle Systems in a Rotating Magnetic Field
}

\author{
S. I. Denisov, ${ }^{1,2}$ T. V. Lyutyy, ${ }^{2}$ and P. Hänggi ${ }^{1}$ \\ ${ }^{1}$ Institut für Physik, Universität Augsburg, Universitätsstraße 1, D-86135 Augsburg, Germany \\ ${ }^{2}$ Sumy State University, 2 Rimsky-Korsakov Street, 40007 Sumy, Ukraine
}

(Received 20 February 2006; published 29 November 2006)

\begin{abstract}
The investigation of a sizable thermal enhancement of magnetization is put forward for uniaxial ferromagnetic nanoparticles that are placed in a rotating magnetic field. We elucidate the nature of this phenomenon and evaluate the resonant frequency dependence of the induced magnetization. Moreover, we reveal the role of magnetic dipolar interactions, point out potential applications, and reason the feasibility of an experimental observation of this effect.
\end{abstract}

DOI: 10.1103/PhysRevLett.97.227202

PACS numbers: 75.50.Tt, 05.40.-a, 75.60.Jk

Presently, the study of magnetic nanoparticles and their structures is one of the most important research areas in nanoscale physics. The first reason is that such nanoparticles increasingly find numerous applications that range from medicine to nanotechnology. Another reason is that these systems exhibit a number of remarkable physical phenomena, such as quantum tunneling of magnetization [1], giant magnetoresistance [2], exchange bias [3], and finite-size and surface effects [4], to name but a few. Moreover, the study of fundamentals of magnetic behavior in these systems is also an important issue, especially for high-density data storage devices [5].

From a practical point of view, the lifetime of stored data and the switching time (i.e., the time during which the reversal of the nanoparticle magnetic moments occurs) are salient characteristics of such devices. Now, thanks to the experimental discovery of fast switching of magnetization [6], the switching time reaches the fundamental (picosecond) limit for field-induced magnetization reversal. On the contrary, a feasible lifetime must cover up to $10 \mathrm{yr}$ and beyond. Its value is usually limited by the superparamagnetic effect [7] and is defined by the probabilities $p_{\sigma}$ that the nanoparticle magnetic moment $\mathbf{m}$ stays in the up ( $\sigma=$ $+1)$ and down $(\sigma=-1)$ equilibrium directions. These probabilities, which are also responsible for other thermal effects in such systems including magnetic relaxation [8], are very sensitive to small perturbations that change the static states of the magnetic moments. Namely, according to the Arrhenius law [9] the ratio $p_{+1} / p_{-1}$ is approximately given by $\exp (\Delta E / k T)$, where $\Delta E=E_{+1}-E_{-1}$, $E_{\sigma}$ is the potential barrier for the reorientation $\sigma \rightarrow-\sigma, k$ is the Boltzmann constant, and $T$ is the absolute temperature. Therefore, if without perturbations $\Delta E=0$, then $p_{+1} / p_{-1}=1$ and the nanoparticle system is demagnetized. But due to the exponential dependence on $\Delta E$ and $T$, the ratio $p_{+1} / p_{-1}$ can drastically be changed by small perturbations. In particular, a static magnetic field $H$ applied along the nanoparticle easy axis of magnetization yields $\Delta E=2 H m(m=|\mathbf{m}|)$, and so $p_{+1}$ strongly differs from $p_{-1}$ if $|H| / H_{a} \gg 1 / 4 a$, where $a=H_{a} m / 2 k T$ and $H_{a}$ is the anisotropy field. This means that even small magnetic fields (in comparison with $H_{a}$ ) almost fully magnetize the nanoparticle systems when $a \gg 1$.

In the case of time-periodic perturbations the situation is not settled yet and far less researched. On the one hand, these perturbations generate dynamical states of the nanoparticle magnetic moments that, because of their natural precession, are expected to be different for the up and down magnetic moments. Therefore, a dynamical magnetization of the system, i.e., a magnetization which is induced by periodic perturbations at $T=0$, can exist but it is expected to be small and, at first sight, it cannot be changed by thermal fluctuations since these perturbations do not change the above mentioned potential barriers. On the other hand, it may be expected that, due to the different precessional states of the up and down magnetic moments, the probabilities $p_{\sigma}$ are different and thus thermal fluctuations contribute to the dynamical magnetization. In this Letter, we attempt to solve this challenge which comprises both basic and applied aspects of the concept of a mean first-passage time.

Dynamical magnetization. - To calculate the dynamical magnetization, we consider the case of identical, noninteracting nanoparticles whose easy axes of magnetization are parallel to each other and the dynamics of the magnetic moment $\mathbf{m}$ is governed by the deterministic LandauLifshitz equation [10]

$$
\dot{\mathbf{m}}=-\gamma \mathbf{m} \times \mathbf{H}_{\mathrm{eff}}-\frac{\lambda \gamma}{m} \mathbf{m} \times\left(\mathbf{m} \times \mathbf{H}_{\mathrm{eff}}\right) .
$$

Here $\gamma(>0)$ is the gyromagnetic ratio, $\lambda(>0)$ is the dimensionless damping parameter, $\mathbf{H}_{\text {eff }}=-\partial W / \partial \mathbf{m}$ is the effective magnetic field acting on $\mathbf{m}$, and $W$ is the nanoparticle magnetic energy. If the easy axes are parallel to the $z$ axis and the external magnetic field $\mathbf{h}(t)$ is circularly polarized in the $x y$ plane, i.e., $\mathbf{h}(t)=$ $h(\cos \omega t, \rho \sin \omega t, 0)$, where $h=|\mathbf{h}(t)|, \omega$ is the frequency of field rotation, and $\rho=-1$ or +1 (the sign - corresponds to the clockwise rotation of $\mathbf{h}(t)$ and the sign + to the counterclockwise one), then

$$
W=\frac{1}{2} m H_{a} \sin ^{2} \theta-m h \sin \theta \cos \psi
$$


( $\psi=\varphi-\rho \omega t$, and $\theta$ and $\varphi$ are the polar and azimuthal angles of $\mathbf{m}$, respectively). For this case, the solution of Eq. (1) is well studied in the context of ferromagnetic resonance [11] and nonlinear magnetization dynamics [12]. Specifically, the precession angles $\theta_{\sigma}$ of the up and down magnetic moments (see Fig. 1) at $\theta_{\sigma} \ll 1$ are given by

$$
\theta_{\sigma}=\frac{\left(1+\lambda^{2}\right) \gamma h}{\sqrt{\left[\left(1+\lambda^{2}\right) \omega_{r}-\rho \sigma \omega\right]^{2}+\bar{\lambda}^{2} \omega^{2}}},
$$

where $\omega_{r}=\gamma H_{a}$. Note that the angles $\theta_{\sigma}$ exhibit the resonance dependence on $\omega$ only for $\rho \sigma=+1$.

We define the dimensionless magnetization of the nanoparticle system induced by the magnetic field $\mathbf{h}(t)$ as $\mu=$ $(1 / N) \sum_{i=1}^{N} m_{z i} / m$ (the index $i$ labels the nanoparticles, $N \gg 1$ ). If the magnetic anisotropy barrier essentially exceeds the thermal energy, i.e., $a \gg 1$, this definition yields $\mu=(1 / N) \sum_{\sigma} \sigma N_{\sigma} \cos \theta_{\sigma}\left(N_{\sigma}\right.$ is the number of magnetic moments in the state $\sigma, N_{-1}+N_{+1}=N$ ), and so $\mu=\sum_{\sigma} \sigma p_{\sigma} \cos \theta_{\sigma}\left(p_{\sigma}=N_{\sigma} / N\right)$. Assuming $p_{\sigma}=$ $1 / 2$ at $T=0$ (this means that the nanoparticle system is demagnetized if $h=0$ ) and using the condition $\theta_{\sigma} \ll 1$ and Eq. (3), for the dynamical magnetization $\mu_{d}=$ $\left.\mu\right|_{T=0}=\left(\theta_{-1}^{2}-\theta_{+1}^{2}\right) / 4$ we obtain

$$
\mu_{d}=-\rho \frac{\left(1+\lambda^{2}\right) \gamma^{2} h^{2} \omega \omega_{r}}{\left[\left(1+\lambda^{2}\right) \omega_{r}^{2}+\omega^{2}\right]^{2}-4 \omega^{2} \omega_{r}^{2}} .
$$

According to this result, the direction of dynamical magnetization $\mu_{d}$ and the direction of magnetic field rotation follow the left-hand rule (the reason is that the natural precession of the magnetic moments is counterclockwise), and the dependence of $\mu_{d}$ on $\omega$ always has a resonant character with $\max \mu_{d}=\left.\mu_{d}\right|_{\omega=\omega_{m}}$, where $\omega_{m}=$ $\left(\omega_{r} / \sqrt{3}\right)\left[1-\lambda^{2}+2\left(1+\lambda^{2}+\lambda^{4}\right)^{1 / 2}\right]^{1 / 2}$. But the value of $\left.\mu_{d}\right|_{\omega=\omega_{m}}$ is very small, however, because with $\max \theta_{\sigma}=$

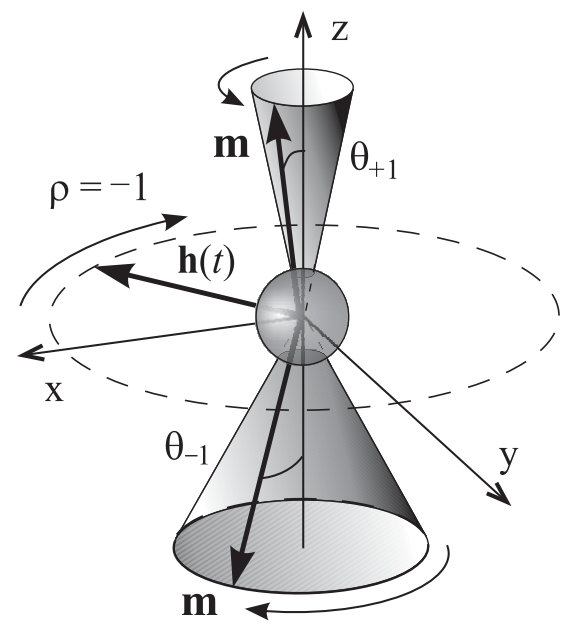

FIG. 1. Sketch of the precession of the up and down magnetic moments (arrows show the directions of their natural precession) at $\rho=-1$. $h / \lambda H_{a}$ even for $\lambda \ll 1$, Eq. (4) yields $\left.\mu_{d}\right|_{\omega=\omega_{m}}=$ $-\rho\left(h / 2 \lambda H_{a}\right)^{2}$. Note also that after switching on the magnetic field $\mathbf{h}(t)$ the initially demagnetized system reaches the steady-state magnetization $\mu_{d}$ during a time interval of the order of $t_{r}=2 / \lambda \omega_{r}$ (we recall that for $T=0$ the states $\sigma$ of the magnetic moments are not changed with time and $\left.p_{\sigma}=1 / 2\right)$.

Thermal enhancement of the dynamical magnetization. - If $T \neq 0$, then the dynamics of the magnetic moments becomes stochastic. In this case, due to thermal fluctuations, the magnetic moments can perform random transitions from the one state $\sigma$ to the other $-\sigma$ and the probabilities $p_{\sigma}$ can thus depend on $\mathbf{h}(t)$. But, in contrast to a static magnetic field, a rotating field has no preferential direction and so it does not impact $p_{\sigma}$ directly. Nevertheless, the probabilities $p_{+1}$ and $p_{-1}$ must be different in the presence of $\mathbf{h}(t)$. The reason is that if the mean times $t_{\sigma}$ which the magnetic moments reside in the states $\sigma$ are much larger than the precession time $2 \pi / \omega$, then the up and down magnetic moments spend almost all time near the conic surfaces with the cone angles $\theta_{+1}$ and $\theta_{-1}$, respectively. Since these angles are different [see Eq. (3)], the times $t_{\sigma}$ must be different as well. Accordingly, because in the steady state $p_{\sigma}=t_{\sigma} / t_{+1}+$ $\left.t_{-1}\right)$, we conclude that the probabilities $p_{\sigma}$ are also different and so thermal fluctuations in fact do contribute to the induced magnetization $\mu$.

Using the conditions $\theta_{\sigma} \ll 1$ and $a \gg 1$, from the definition of $\mu$ we obtain $\mu=\mu_{t}+\mu_{d}$ [we neglect the term $\left.\mu_{t}\left(\theta_{-1}^{2}+\theta_{+1}^{2}\right) / 4\right]$, where $\mu_{t}=p_{+1}-p_{-1}$ is the desired contribution arising from the joint action of thermal fluctuations and rotating field. According to the above argumentation, the condition $p_{\sigma}<p_{-\sigma}$ holds if $\theta_{\sigma}>$ $\theta_{-\sigma}$. This implies that the thermal contribution $\mu_{t}$ always enhances the deterministic part $\mu_{d}$. Moreover, one expects the enhancement increases with decreasing temperature. It is important to note in this context that $\mu$ denotes the equilibrium magnetization which is established during a time interval of the order of the transition time $t_{\text {tr }}$ between the states $\sigma$ and $-\sigma\left(t_{\mathrm{tr}} \sim \max t_{\sigma} \gg t_{r}\right.$ and $t_{\mathrm{tr}} \rightarrow \infty$ as $T \rightarrow 0$ ). At these times the probabilities $p_{+1}$ and $p_{-1}$ are generally different and thus $\lim _{T \rightarrow 0} \mu \neq \mu_{d}$.

For determining the mean residence times $t_{\sigma}$ which define the probabilities $p_{\sigma}$ and the magnetization $\mu_{t}$, we used the mean first-passage time formalism [9,13]. Its application to our situation is well founded because in the case of small rotating field the magnetic moments that are in the state $\sigma$ reach any point of the separatrix, which separates the up and down states, with almost the same probability density. Given that the stochastic dynamics of the magnetic moments is Markovian [14], the standard mean first-passage procedure is employed in order to account for the influence of the rotating field. Specifically, starting out from the two-dimensional backward FokkerPlanck equation $[13,15]$ in the rotating frame, we suc- 
ceeded to derive a mathematically tractable and physically transparent expression for the mean residence times:

$$
t_{\sigma}=t_{0} \exp \left[a\left(-\theta_{\sigma}^{2}+\sigma 2 H_{\text {eff }} / H_{a}\right)\right],
$$

where $t_{0}=t_{r} \sqrt{\pi / 4 a} e^{a}$ is the mean time which the magnetic moment spends in the up or down state at $h=0$, and $H_{\text {eff }}=-\rho p \gamma h^{2} / \omega\left(\left|H_{\text {eff }}\right| \ll H_{a}, p \sim 1\right)$.

As follows from (5), the rotating magnetic field influences the mean residence times $t_{\sigma}$ via two different mechanisms. A first one consists in the appearance of the dynamical states of the magnetic moments which are characterized by the precession angles $\theta_{\sigma}$. The contribution of these states to $t_{\sigma}$ is governed by the first term in the argument of the exponential function, which always decreases $t_{\sigma}$. The second mechanism consists in changing the effective potential barrier between the up and the down states. Its contribution to $t_{\sigma}$ is described by the second term in the argument of the exponential function, where $H_{\text {eff }}$ can be interpreted as a static effective magnetic field applied along the easy axis of magnetization. Since the sign of $H_{\text {eff }}$ depends on $\rho$, this mechanism can either increase or decrease $t_{\sigma}$.

Using Eqs. (3) and (5), the definition $\mu_{t}=\left(t_{+1}-\right.$ $\left.t_{-1}\right) /\left(t_{+1}+t_{-1}\right)$ leads to our main result:

$$
\mu_{t}=\tanh \left[2 a\left(\mu_{d}+H_{\mathrm{eff}} / H_{a}\right)\right] .
$$

It shows that the rotating field always magnetizes the nanoparticle system perpendicular to the plane of field rotation and the direction of magnetization is uniquely defined by the direction of field rotation: $\operatorname{sgn} \mu_{\infty}=-\rho$. In general, both mentioned mechanisms contribute to $\mu_{t}$. However, in the most interesting resonant case, when $\omega \sim$ $\omega_{m}$ and $\lambda \ll 1$, the first mechanism is dominating $\left(\mu_{d} H_{a} / H_{\text {eff }} \sim \lambda^{-2} \gg 1\right)$, and thus the magnetization (6) becomes

$$
\mu_{t}=\tanh \left(2 a \mu_{d}\right) .
$$

According to this relation, being valid for $a \gg 1$ and $\left|\mu_{d}\right| \ll 1$, the condition $\mu_{t} / \mu_{d} \gg 1$ always holds (specifically, if $a\left|\mu_{d}\right| \ll 1$, then $\mu_{t} / \mu_{d} \approx 2 a$ ). This means that a small dynamical magnetization is strongly enhanced by thermal fluctuations, i.e., $\mu \approx \mu_{t}$. Comparing (7) with the magnetization of an Ising paramagnet, $\tanh (m H / k T)$, we see that the magnetic field rotating in a plane perpendicular to the easy axes of the nanoparticles acts as a static magnetic field $H=H_{a} \mu_{d}$, which is applied along these axes. As in the case with $\mu_{d}$, the induced magnetization $\mu_{t}$ as a function of $\omega$ exhibits a resonance character. The dependence of $\mu_{t}$ on the reduced frequency $\tilde{\omega}=\omega / \omega_{r}$ is depicted in Fig. 2, curve 1, for a system of spherical nanoparticles with $H_{a}=6400 \mathrm{Oe}, m / V=1400 \mathrm{G}(V$ is the nanoparticle volume), $r=4 \mathrm{~nm}$ ( $r$ is the nanoparticle radius), $\lambda=10^{-2}, h=10 \mathrm{Oe}, \rho=-1$, and $T=300 \mathrm{~K}$. Note that for these parameters $a=H_{a} m / 2 k T \approx 29$, $\left.\mu_{t}\right|_{\tilde{\omega}=1} \approx 0.34,\left.\mu_{d}\right|_{\tilde{\omega}=1} \approx 6.1 \times 10^{-3}$, and $\mu_{t} / \mu_{d} \approx 56$.

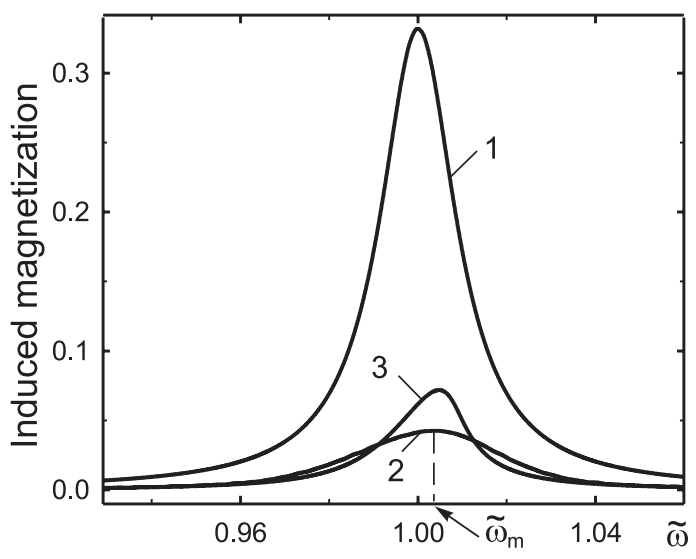

FIG. 2. Plots of $\mu_{t}$ (curve 1), $\left\langle\mu_{t}\right\rangle$ (curve 2), and $\mu_{t}^{\mathrm{mf}}$ (curve 3) as the functions of the reduced frequency $\tilde{\omega}$.

Thus, the above results show that the magnetic field rotating in the plane perpendicular to the easy axes of magnetic nanoparticles changes the probabilities of the up and down orientations of the nanoparticle magnetic moments. Because of the thermal fluctuations, the nanoparticle system magnetizes in the direction with larger probability. In the case of nanoparticles with large anisotropy barrier (when $a \gg 1$ ) this contribution, i.e., $\mu_{t}$, to the total magnetization $\mu$ considerably exceeds a small dynamical contribution, i.e., $\mu_{d}$, which is induced by the rotating magnetic field at $T=0$. We emphasize that the magnetization of nanoparticle systems in the rotating magnetic field arises from the different dynamical behavior of the magnetic moments in the up and down states. In turn, the difference in the dynamics of the magnetic moments results from the existence of a well-defined direction of their natural precession (counterclockwise when viewed from above).

Role of dipolar interactions. - To check the role of the magnetic dipolar interaction, we performed a Monte Carlo simulation for two-dimensional arrays of dipolar interacting nanoparticles, representing an important class of patterned magnetic recording media [5]. In doing so we assumed that the centers of $N$ nanoparticles occupy the sites of a square lattice of size $L d \times L d$ ( $L$ is a natural number, $(L+1)^{2}=N$, and $d$ is the lattice spacing). The easy axes of the nanoparticles are perpendicular to the lattice plane and the magnetic field rotates in this plane. In contrast to the previous case, the dipolar magnetic field acts on each magnetic moment. This field is changed from site to site and, due to the random motion of the magnetic moments, fluctuates with time. For $a \gg 1$, the fluctuations of the magnetic moments and the rates of their reorientations are small. Thus, the dipolar field acting on the $i$ th magnetic moment during the $l$ th step can be approximated as $\mathbf{H}_{i}(l)=\left[0,0, H_{i}(l)\right]$, where $H_{i}(l)=-m \sum_{j \neq i} \sigma_{j}(l) / r_{i j}^{3}$, $\sigma_{j}(l)=+1$ or -1 , and $r_{i j}$ is the distance between the centers of the nanoparticles. In this case, the magnetization 
of the nanoparticle system can be represented through the step-dependent magnetization $\mu_{t}(l)=\sum_{i=1}^{N} \sigma_{i}(l) / N$ as follows:

$$
\left\langle\mu_{t}\right\rangle=\frac{1}{l_{2}-l_{1}+1} \sum_{l=l_{1}}^{l_{2}} \mu_{t}(l) .
$$

Here, to be sure that the system reached the steady state and the averaging procedure is correct, the conditions $l_{1} \gg$ 1 and $l_{2}-l_{1} \gg 1$ are implied.

In order to apply the Monte Carlo method for calculating $\left\langle\mu_{t}\right\rangle$, we need to evaluate for all $i$ and $l$ the probabilities $p_{\sigma_{i}}(i, l)$ that the $i$ th magnetic moment stays in the states $\sigma_{i}(l)$. But in our case the conventional approach involving the Boltzmann factor for the solution of this problem is not applicable because the rotating field depends on time. Therefore, we extended the above method to the case of dipolar interacting nanoparticles and calculated the mean times that the $i$ th magnetic moment spends in the up and down states:

$$
t_{\sigma_{i}}=t_{0} \frac{\exp \left[a\left(\cos \theta_{\sigma_{i}}+\sigma_{i} b_{i}\right)^{2}-a\right]}{\left(1-b_{i}^{2}\right)\left(\cos \theta_{\sigma_{i}}+\sigma_{i} b_{i}\right)},
$$

where $b_{i}=H_{i}(l) / H_{a}$ and $\theta_{\sigma_{i}}$ is the precession angle of the $i$ th magnetic moment that is defined by Eq. (3) in which $\sigma$ should be replaced by $\sigma_{i}(l)$ and $\omega_{r}$ by $\omega_{r}+\gamma H_{i}(l)$ (for brevity, we omitted the arguments $i$ and $l$ ). Next, defining $p_{\sigma_{i}}(i, l)=t_{\sigma_{i}}(i, l) /\left[t_{+1}(i, l)+t_{-1}(i, l)\right]$ and using the numerical procedure developed in [16], we performed a Monte Carlo simulation of the magnetization $\left\langle\mu_{t}\right\rangle$ induced by the rotating field in two-dimensional systems of dipolar interacting nanoparticles.

In Fig. 2, curve 2, we depict the dependence of $\left\langle\mu_{t}\right\rangle$ on $\tilde{\omega}$ for the square array of the same nanoparticles driven by the same rotating field at $N=10^{4}, d=5 r, l_{1}=10^{3}$, and $l_{2}=$ $5 \times 10^{3}$. Comparing curves 1 and 2 shows that the magnetic dipolar interaction reduces the induced magnetization, widens its frequency dependence, and raises the resonance frequency $\left(\tilde{\omega}_{m}>1\right)$. We emphasize that although the dipolar interaction reduces the induced magnetization, its experimental observation is still possible even in this strongly interacting case. Note also that $\mu_{t}^{\mathrm{mf}}$, which we evaluated within the mean-field approximation (see Fig. 2, curve 3), distinctly differs from $\left\langle\mu_{t}\right\rangle$ because this approximation does not account for the crucial feature of the dipolar interaction in these systems, i.e., its antiferromagnetic character.

Potential applications. - The above results evidence that the frequency dependence of the induced magnetization is detectable, and because the magnetic resonance methods are both very accurate and sensitive, its experimental determination thus provides valuable information about the dipolar field distribution in such systems. In particular, the average dipolar field acting on the resonant particles can approximately be estimated as $\rho H_{a}\left(\tilde{\omega}_{m}-1\right)$. Moreover, due to the selective change of the thermal stability of the magnetic moments, which is controlled by the characteristics of the rotating magnetic field, it calls for potential applications in magnetic recording technology.

Resume. - We succeeded to show that a small dynamical magnetization of nanoparticle systems that is induced by the circularly polarized magnetic field is strongly enhanced by thermal fluctuations. The thermally enhanced magnetization exhibits as a function of the field frequency a resonant character, possessing a well-pronounced extremum. The magnetic dipolar interaction increases the relative width of the frequency dependence of magnetization while causing a decrease of its strength and a corresponding shift of its maximum to higher frequencies.

S.I.D. and T. V.L. acknowledge the support of the EU through NANOSPIN Contract No. NMP4-CT-2004013545, S.I.D. acknowledges the support of EU Contract No. MIF1-CT-2005-007021, and P. H. acknowledges the support of the DFG via the SFB 486.

[1] Quantum Tunneling of Magnetization-QTM'94, edited by L. Gunther and B. Barbara (Kluwer, Dordrecht, 1995); E. M. Chudnovsky and J. Tejada, Macroscopic Quantum Tunneling of the Magnetic Moment (Cambridge University Press, Cambridge, 1998).

[2] A. E. Berkowitz et al., Phys. Rev. Lett. 68, 3745 (1992); J. Q. Xiao, J.S. Jiang, and C. L. Chien, ibid. 68, 3749 (1992).

[3] For a recent review, see J. Nogués et al., Phys. Rep. 422, 65 (2005).

[4] Surface Effects in Magnetic Nanoparticles, edited by D. Fiorani (Springer, Berlin, 2005).

[5] C. A. Ross, Annu. Rev. Mater. Res. 31, 203 (2001); B. D. Terris and T. Thomson, J. Phys. D 38, R199 (2005).

[6] C. H. Back et al., Phys. Rev. Lett. 81, 3251 (1998); H. W. Schumacher et al., ibid. 90, 017201 (2003).

[7] L. Néel, Ann. Geophys. 5, 99 (1949).

[8] For a comprehensive review, see J. L. Dorman, D. Fiorani, and E. Tronc, Adv. Chem. Phys. 98, 283 (1997), and references therein.

[9] P. Hänggi, P. Talkner, and M. Borkovec, Rev. Mod. Phys. 62, 251 (1990).

[10] L. Landau and E. Lifshitz, Phys. Z. Sowjetunion 8, 153 (1935).

[11] See, for example, A. G. Gurevich and G. A. Melkov, Magnetization Oscillations and Waves (CRC Press, Boca Raton, 1996), and references therein.

[12] G. Bertotti, C. Serpico, and I. D. Mayergoyz, Phys. Rev. Lett. 86, 724 (2001).

[13] P. Hänggi and H. Thomas, Phys. Rep. 88, 207 (1982).

[14] W. F. Brown, Jr., Phys. Rev. 130, 1677 (1963).

[15] S.I. Denisov and A. N. Yunda, Physica (Amsterdam) 245B, 282 (1998).

[16] S. I. Denisov, T. V. Lyutyy, and K. N. Trohidou, Phys. Rev. B 67, 014411 (2003). 